\title{
White Matter Hyperintensity in Ischemic Stroke Patients: It May Regress Over Time
}

\author{
A-Hyun Cho, ${ }^{a}$ Hyeong-Ryul Kim, ${ }^{\mathrm{b}}$ Woojun Kim, ${ }^{\mathrm{a}}$ Dong Won Yang ${ }^{\mathrm{c}}$ \\ ${ }^{a}$ Department of Neurology, College of Medicine, The Catholic University of Korea, Yeouido St. Mary's Hospital, Seoul, Korea \\ b'Department of Preventive Medicine, College of Medicine, The Catholic University of Korea, Seoul St. Mary's Hospital, Seoul, Korea \\ 'Department of Neurology, College of Medicine, The Catholic University of Korea, Seoul St. Mary's Hospital, Seoul, Korea
}

Background and Purpose White matter hyperintensities (WMH) are frequently observed on MRI in ischemic stroke patients as well as in normal elderly individuals. Besides the progression of $\mathrm{WMH}$, the regression of WMH has been rarely reported. Thus, we aimed to investigate how WMH change over time in patients with ischemic stroke, particularly focusing on regression.

Methods We enrolled ischemic stroke patients who underwent brain MRI more than twice with at least a 6 month time-interval. Based on T2-weighted or FLAIR MRI, WMH were visually assessed, followed by semiautomatic volume measurement. Progression or regression of WMH change was defined when $0.25 \mathrm{cc}$ increase or decrease was observed and it was also combined with visible change. A statistical analysis was performed on the pattern of WMH change over time and factors associated with change.

Results A total of 100 patients were enrolled. Their age (mean \pm SD) was $67.5 \pm 11.8$ years and 63 were male. The imaging time-interval (mean) was 28.0 months. WMH progressed in 27, regressed in 9 and progressed in distinctive regions and regressed in others in 5 patients. A multiple logistic regression model showed that age (odds ratio[OR] 2.51, 90\% confidence interval[Cl] 1.056-5.958), male gender (OR 2.957, 95\% Cl 1.051-9.037), large vessel disease (OR 1.955, 95\% Cl 1.171-3.366), and renal dysfunction (OR 2.900, 90\% Cl 1.045-8.046) were associated with progression. Regarding regression, no significant factor was found in the multivariate analysis.

Conclusions In 21.5\% of ischemic stroke patients, regression of WMH was observed. WMH progression was observed in a third of ischemic stroke patients.

Keywords Ischemic stroke; White matter hyperintensity; Leukoaraiosis; MRI, progression; Regression

\author{
Correspondence: A-Hyun Cho \\ Department of Neurology, College of \\ Medicine, The Catholic University of \\ Korea, Yeouido St. Mary's Hospital, \\ 10 63-ro, Yeongdeungpo-gu, Seoul \\ 150-713, Korea \\ Tel: $+82-2-3779-2433$ \\ Fax: +82-2-782-8654 \\ E-mail: ahyun@catholic.ac.kr
}

Received: November 15, 2014

Revised: January 5, 2015

Accepted: January 7, 2015

\begin{abstract}
This research was supported by Basic Science Research Program through the National Research Foundation of Korea (NRF) funded by the Ministry of Education, Science and Technology (No.2012R1A1B5000477). All authors appreciate the support from Korean Neurological Association (Myeong-In Academy Awards).
\end{abstract}

The authors have no financial conflicts of interest.

\section{Introduction}

White matter hyperintensities (WMH) are frequently observed on T2- or fluid-attenuated inverse recovery (FLAIR) MRI in ischemic stroke patients as well as in normal elderly individuals. WMH is also described as leukoaraiosis, meaning neuroimaging abnormalities of the white matter that are seen as bilaterally patch or dif- fuse areas of hypo-density on CT or hyperintensity on T2-weighted or FLAIR MRI.

More than half of all elderly individuals have some degree of white matter lesions on MRI. ${ }^{1}$ However, the exact pathophysiology and their clinical significance are still unclear. A recent review article suggested that $\mathrm{WMH}$ predict an increased risk of stroke, dementia, and death, supporting their use as an intermediate 
marker for research. ${ }^{2}$ The probable pathogenesis reported up to now has mostly involved ischemic pathogenesis. In addition, alternative hypotheses such as disturbance of cerebrospinal fluid circulation and cerebral edema from altered blood-brain barrier have also been raised. Therefore, we hypothesized that the change of WMH over time may be dynamic. In other words, they may not only progress but also regress.

Previous studies on WMH change were mostly about progression in community-dwelling subjects, not about regression. 8.1\%$28 \%$ of non-demented elderly patients showed WMH progression with 2-6 year intervals. ${ }^{3}$ The predictors of $\mathrm{WMH}$ worsening were smoking, infarct or WMH on the initial scan, old age, high blood pressure, and diabetes. ${ }^{4,5}$ However, the reports on regression of WMH are sparse and have been presented as case reports. ${ }^{6-9}$

In this context, we aimed to investigate how WMH regress or progress over time and to determine factors related to changes particularly in ischemic stroke patients.

\section{Methods}

\section{Patients}

We retrospectively enrolled ischemic stroke patients who underwent brain MRI (including T2-weighted image or FLAIR image) more than twice with at least a 6 month-time-interval. Ischemic stroke was diagnosed by a stroke physician with confirmation based on diffusion-weighted MRI (DWI). Patients with ischemic pathologic lesions (index stroke) which could not be distinguished from WMH were excluded from the study.

\section{MRI protocols and volume measurement}

The sequence (T2 weighted- or FLAIR) of the initial MRI scan and that of the follow-up scan in a patient should be the same. The common MRI parameters for DWI, FLAIR, T2-weighted images were a slice thickness of $45 \mathrm{~mm}$, an inter-slice gap of $2 \mathrm{~mm}$, and 22 axial slices. DWI parameters included a field-of-view of $240 \mathrm{~mm}$, a repetition time (TR) of $7,000 \mathrm{msec}$, an echo time (TE) of $85.3 \mathrm{msec}$, a matrix number of $128 \times 128$, and two b values of 0 and $1,000 \mathrm{sec} /$ $\mathrm{mm}^{2}$. FLAIR imaging was obtained using a fast-spin echo sequence having TR/TE $=9,902 / 125.8 \mathrm{msec}$, inversion time $=2,400 \mathrm{msec}$, a field-of-view of $220 \mathrm{~mm}$ and a $320 \times 256$ matrix. The TR/TE of T2Weighted image was 4,000/100.3 msec. Based on T2-weighted or FLAIR MRI, WMH was visually assessed (modified Fazekas grading), ${ }^{10}$ and semiautomatic volume measurement was performed by an independent investigator masked to clinical data. Semoautomatic volume measurement was done by ANALYZE 10.0. ${ }^{11}$ FLAIR and T2 images with DICOM format were loaded. Inhomogeneity correction was done and skull bone was removed manually. We analyzed histogram of voxel intensities and decided threshold of
WMH visually. Region of interest (ROI) was selected with threshold and was corrected manually. Volume of ROI was calculated. WMH were not measured when it is probably located within acute ischemic lesions. A change of WMH was interpreted as 1) no change, 2) progression, 3 ) regression, and 4) both progression and regression. The definition of both progression and regression was done when progression was found in one region and regression was found in another region in the same patient.

Progression or regression of WMH change was defined when more than $0.25 \mathrm{cc}$ increase or decrease was observed with significant visual change. The $0.25 \mathrm{cc}$ was the smallest volume change that was also interpreted as progression or regression by visual assessment. The definite visible change (progression or regression) was determined by two independent raters (A. H. Cho, W. J. Kim). Although we assessed WMH with modified Fazekas grading initially, the minor WMH change was not interpreted with use of the grading system, because minor but significant change of WMH could not affect Fazekas grading score. The inter-observer agreement (kappa value) was 0.884 and 0.912 for progression and regression. In cases with both progression and regression, the numerical volume data could not be considered as decision criteria because both the volume increase and decrease are simultaneously present. In this case, we interpreted the data entirely dependent on detailed visual assessment.

\section{Clinical data}

Clinical variables (age, sex, risk factors, stroke subtypes, blood pressure, creatinine, glomerular filteration rate (GFR), hemoglobin, hsCRP and imaging time-interval) were obtained by reviewing medical records. Stroke subtypes were classified according to the modified TOAST subtype classification. ${ }^{12}$ Blood pressure was checked at the time of the first scan and follow-up scan. Serum creatinine level and GFR were obtained within 3 months after an incidence of stroke.

\section{Data analysis}

A descriptive analysis was done on the pattern of WMH change over time, and possible factors associated with each changing pattern were analyzed. To compare baseline characteristics between patients with progression and those without, we used Student's $t$ test for age and time interval, Mann Whitney U test for hsCRP, Chi-square test for sex, hypertension, diabetes, hyperlipidemia, and smoking, and Fisher exact test for TOAST subtype. Regarding regression, Student's $t$ test (age, time interval), Mann Whitney $\mathrm{U}$ test (hsCRP), the Chi-square test (sex, hyperlipidemia), and Fisher exact test (hypertension, smoking, TOAST subtype) were used. In the multiple logistic regression model, factors with $P<0.3$ according to univariate analysis were entered. SAS 
9.2 version was used. This study was approved by institutional review board of our hospital. An informed consent was not obtained because this study is retrospectively performed.

\section{Results}

\section{Baseline characteristics}

Between November 2010 and May 2012, a total of 100 patients were enrolled. Reasons for follow-up MRI were neurologic symptoms $(n=31)$, routine check-up $(n=12)$ and other research enrollment $(n=57)$. Their age $($ mean \pm SD $)$ was $67.5 \pm$ 11.8 years and 63 were male. The most common stroke subtype was small vessel disease (SVD, 43\%), followed by large vessel disease (LVD, 27\%) and cardioembolism (9\%). The imaging time-interval (mean \pm SD) was $28.0 \pm 16.6$ months. Imaging analysis was done with T2-weighted MRI in 42 patients and FLAIR MRI in 58 patients. The proportion of progression and regression was not affected by the T2 or FLAIR imaging method $(P=0.80, P=0.60)$.

\section{White matter hyperintensity on MRI}

On the initial MRI scan, WMH was observed in 65 patients. During the follow-up period, 27 patients showed progression of WMH (Figure 1) and 9 showed regression (Figures 2, 3). In 5 patients, both progression and regression were observed. Table 1 shows the clinical characteristics of patients with regression of WMH. Three patients among those without WMH on the initial scan had newly developed WMH on the follow-up scan. The initial WMH volume (median and interquartile range) was $2.78 \mathrm{cc}(0.77-11.57)$. The volume difference in patients with
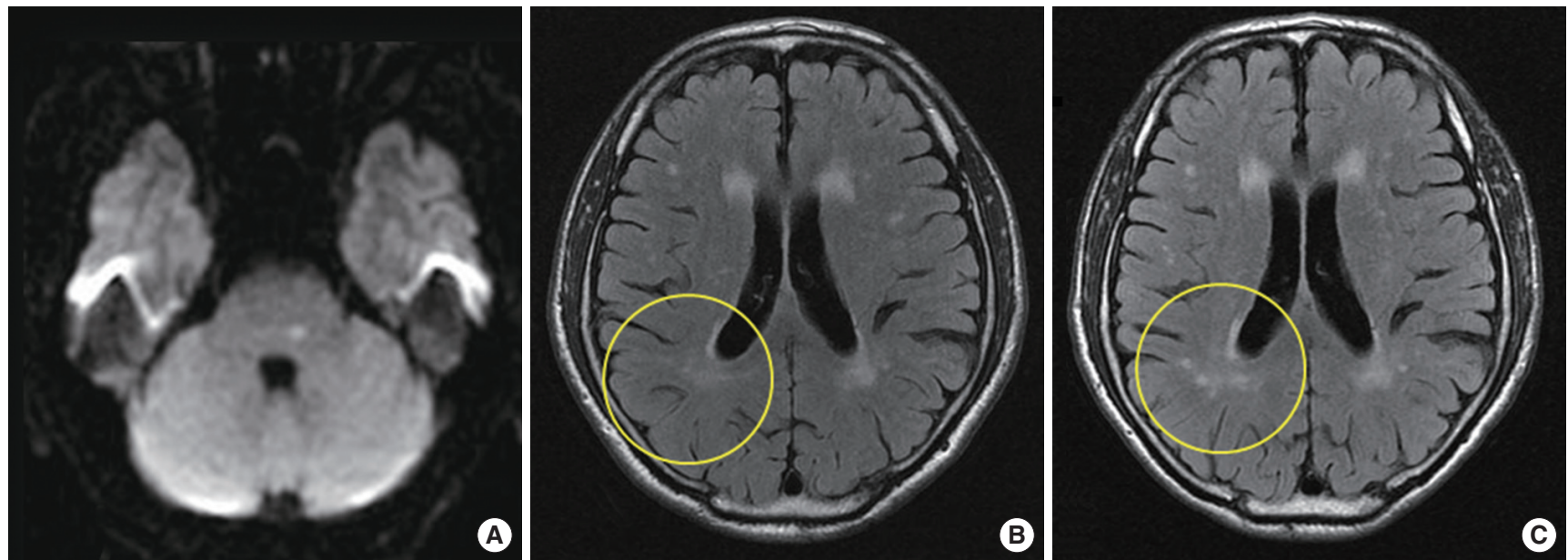

Figure 1. A 77-year-old female presented with left pontine infarction (A). Progression of white matter hyperintensity is observed on FLAIR MRI at 3.5 years of followup (B, initial scan; $\mathrm{C}$, follow-up).
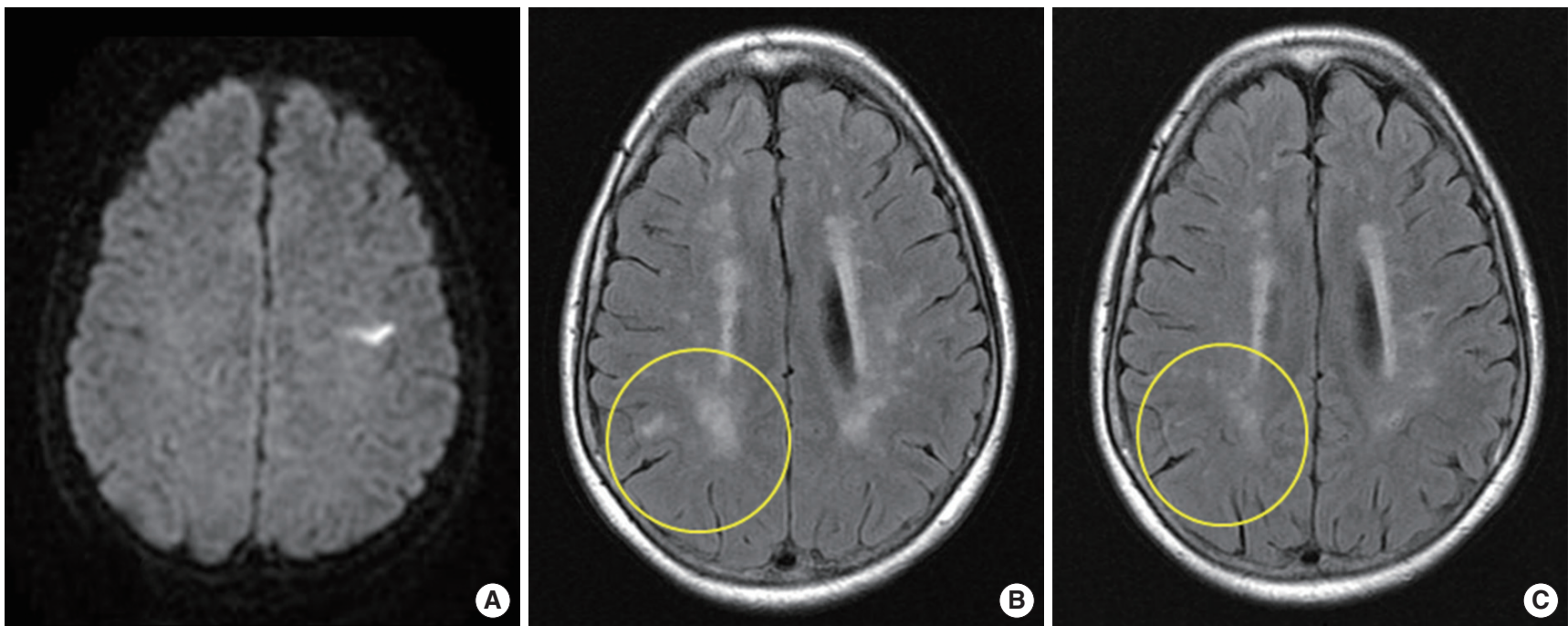

Figure 2. A 68-year-old female presented with left MCA territorial infarction (A). Regression of white matter hyperintensity is observed on FLAIR at 21 months of follow-up (B, initial scan; C, follow-up). 

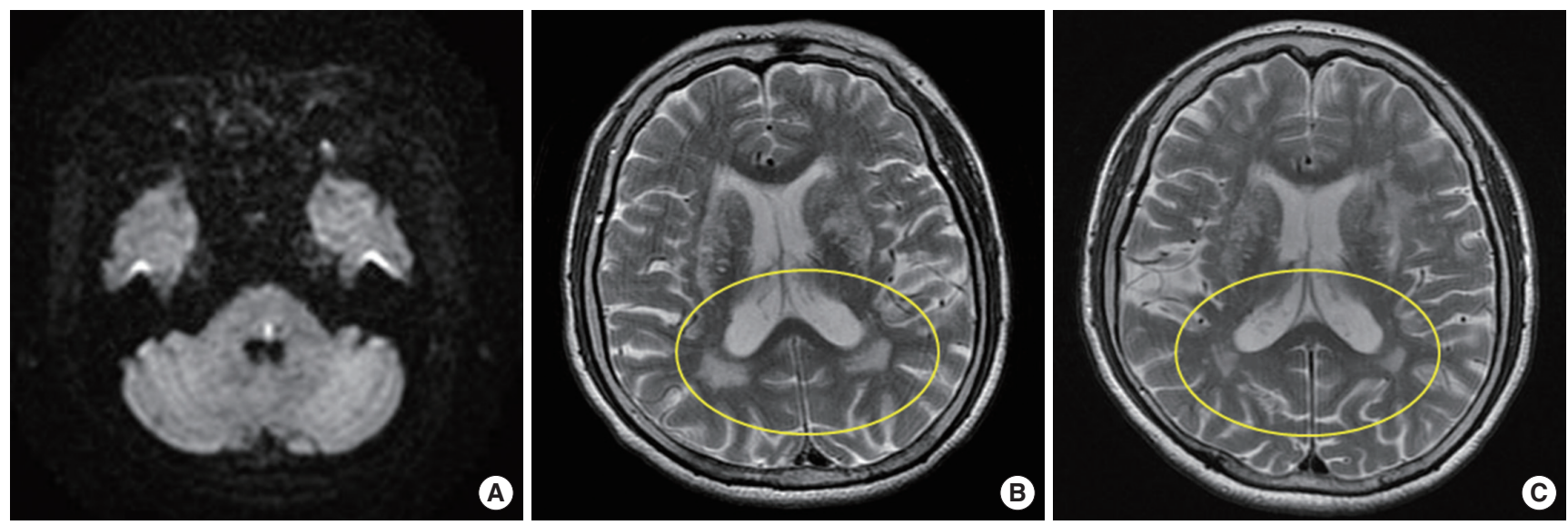

Figure 3. A 72-year-old female presented with left pontine infarction (A). Regression of white matter hyperintensity is observed on T2-weighted MRI at 7 months of follow-up (B, initial scan; C, follow-up).

Table 1. Clinical and radiological characteristics of patients with regression of white matter hyperintensity

\begin{tabular}{|c|c|c|c|c|c|c|c|c|c|c|}
\hline \multirow{2}{*}{ No. } & \multirow{2}{*}{$\begin{array}{l}\text { Age } \\
\text { /sex }\end{array}$} & \multirow{2}{*}{$\begin{array}{l}\text { Acute ischemic } \\
\text { lesion }\end{array}$} & \multirow{2}{*}{$\begin{array}{l}\text { Clinical } \\
\text { Symptom }\end{array}$} & \multirow{2}{*}{$\begin{array}{c}\text { Stroke } \\
\text { mechanism }\end{array}$} & \multirow{2}{*}{$\begin{array}{l}\text { Medical } \\
\text { illness }\end{array}$} & \multirow{2}{*}{ Large artery } & \multicolumn{2}{|c|}{ Volume (cc) } & \multirow{2}{*}{$\begin{array}{l}\text { Interval } \\
\text { (month) }\end{array}$} & \multirow{2}{*}{ Regression site } \\
\hline & & & & & & & Initial & Second & & \\
\hline 1 & 70/M & Ltpons & $\begin{array}{r}\text { Facial palsy, } \\
\text { dysarthria }\end{array}$ & CE & HT, DM, HL & Rt MCA focal stenosis & 5.97 & 4.44 & 18 & $\begin{array}{l}\text { Periventricular white matter, bilateral } \\
\text { posterior horn }\end{array}$ \\
\hline 2 & 79/M & Lt pons & Dysarthria & SVD & HT & Normal & 6.07 & 3.12 & 29 & Lt parietal subcortical \\
\hline 3 & $70 / F$ & Lt frontal lobe & Aphasia & LVD & $H T, H L$ & $\begin{array}{l}\text { Lt MCA, PCA, BA } \\
\text { stenoses }\end{array}$ & 106.78 & 101.30 & 46 & $\begin{array}{l}\text { Periventricular white matter, bilateral } \\
\text { posterior horn }\end{array}$ \\
\hline 4 & $80 / F$ & Lt. frontal & Dysarthria & LVD & HT, smoking & Lt MCA stenosis & 6.43 & 4.31 & 25 & Rt subcortical \\
\hline 5 & $77 / \mathrm{M}$ & Rt pons & Lt hemiparesis & SVD & HT, DM & Normal & 0.72 & 1.31 & 18 & $\begin{array}{l}\text { Lt frontal, Rt parietal subcortical } \\
\text { (c progression) }\end{array}$ \\
\hline 6 & $77 / F$ & Lt. internal capsule & Rt hemiparesis & SVD & HT, DM, HL & Normal & 25.57 & 28.82 & 27 & Lt. frontal subcortical (c progression) \\
\hline 7 & $73 / F$ & Lt pontomedulla & Rt hemiparesis & SVD & HT, DM & Rt distal ICA stenosis & 15.93 & 10.79 & 7 & $\begin{array}{l}\text { Periventricular white matter, bilateral } \\
\text { posterior horn }\end{array}$ \\
\hline 8 & $60 / M$ & Rt MCA cortex & Lt arm weakness & Cryptogenic & Smoking & Normal & 0.75 & 0.24 & 26 & Bilateral centrum semiovale \\
\hline 9 & $68 / F$ & Lt MCA cortex & Dysarthria & Cryptogenic & & Normal & 20.29 & 13.26 & 14 & $\begin{array}{l}\text { Bilateral parietal subcortical } \\
\text { (c progression) }\end{array}$ \\
\hline 10 & $52 / \mathrm{M}$ & Lt internal capsule & Rt hemiparesis & SVD & HT, smoking & Both VA stenoses & 2.11 & 0.94 & 21 & Rt parietal, Lt corona radiata \\
\hline 11 & $80 / \mathrm{M}$ & Lt pons & Dysarthria & SVD & HT & Normal & 10.43 & 6.24 & 48 & $\begin{array}{l}\text { Bilateral posterior horn } \\
\text { (c progression) }\end{array}$ \\
\hline 12 & $77 / F$ & Rt pons & Lt hemiparesis & SVD & HT, DM & Both ICA stenoses & 9.79 & 5.17 & 48 & Bilateral posterior horn \\
\hline 13 & $49 / M$ & Lt pons & Dysarthria & LVD & HT, DM, HL & BA stenosis & 3.46 & 1.83 & 13 & $\begin{array}{l}\text { Both frontal subcortical } \\
\text { (c progression) }\end{array}$ \\
\hline 14 & $52 / \mathrm{M}$ & $\begin{array}{l}\text { Rt thalamus, } \\
\text { Lt temporal }\end{array}$ & Lt hemisensory loss & Cryptogenic & HT, smoking & Normal & 6.10 & 5.36 & 15 & Rt parietal subcortical \\
\hline
\end{tabular}

$\mathrm{CE}$, cardioembolism; HT, hypertension; DM, diabetes mellitus; HL, hyperlipidemia; MCA, middle cerebral artery; ICA, internal carotid artery; VA, vertebral artery; BA, basilar artery.

progression was $3.32 \mathrm{cc}(0.92-4.05)$ and that in patients with regression was $-1.87 \mathrm{cc}(-4.61--0.73)$.

\section{Factors associated with WMH change}

We could not find any significantly associated factor with regression of WMH in this study. Regarding progression, univariate analysis showed that old age, large vessel disease, and time interval were associated with progression $(P=0.023,0.015$, 0.018 , respectively, Table 2). After adjustment for time interval, the multiple logistic regression model revealed that age (odds ratio $[\mathrm{OR}] 2.508,90 \%$ confidence interval $[\mathrm{CI}]$ 1.056-5.958), male gender (OR 2.957, 95\% CI 1.051-9.037), large vessel disease (OR 1.955, 95\% CI 1.171-3.366), and poor renal function (OR 2.900, 90\% CI 1.045-8.046) were factors associated with progression (Table 3 ).

\section{Discussion}

We firstly investigated the changing pattern of coexisting white matter change (leukoaraiosis) over time in ischemic 
Table 2. Comparison of baseline characteristics in patients with white matter hyperintensities change and those without

\begin{tabular}{|c|c|c|c|c|c|c|}
\hline & \multicolumn{2}{|c|}{ Progression } & \multirow{2}{*}{$P$} & \multicolumn{2}{|c|}{ Regression } & \multirow{2}{*}{$P$} \\
\hline & Yes $(n=32)$ & No $(n=68)$ & & Yes $(n=14)$ & No $(n=51)$ & \\
\hline Age (mean \pm SD) & $71.4 \pm 11.9$ & $65.7 \pm 11.3$ & 0.023 & $68.9 \pm 11.1$ & $70.7 \pm 11.2$ & 0.660 \\
\hline Sex (male) & $23,71.9 \%$ & $40,58.8 \%$ & 0.268 & $8,57.1 \%$ & $33,64.7 \%$ & 0.603 \\
\hline HTN (n, \%) & $23,71.9 \%$ & $50,73.5 \%$ & $>0.999$ & $12,85.7 \%$ & $37,72.5 \%$ & 0.487 \\
\hline $\mathrm{DM}(\mathrm{n}, \%)$ & $14,43.8 \%$ & $24,35.3 \%$ & 0.509 & $6,42.9 \%$ & $22,43.1 \%$ & 0.985 \\
\hline$H L(n, \%)$ & $17,53.1 \%$ & $27,39.7 \%$ & 0.280 & $4,28.6 \%$ & $23,45.1 \%$ & 0.266 \\
\hline Smoking (n, \%) & $4,12.5 \%$ & $19,27.9 \%$ & 0.126 & $4,28.6 \%$ & $9,17.6 \%$ & 0.453 \\
\hline \multicolumn{7}{|l|}{ TOAST } \\
\hline $\operatorname{SVD}(n=43)$ & $13,40.6 \%$ & $30,44.1 \%$ & 0.028 & $7,50.0 \%$ & $21,41.2 \%$ & 0.736 \\
\hline $\operatorname{LVD}(n=27)$ & $14,43.8 \%$ & $13,19.1 \%$ & & $3,21.4 \%$ & $18,35.3 \%$ & \\
\hline $\mathrm{CE}(\mathrm{n}=9)$ & 0 & $9,13.2 \%$ & & $1,7.1 \%$ & $2,3.9 \%$ & \\
\hline Undetermined $(n=17)$ & $3,9.3 \%$ & $14,20.6 \%$ & & $3,21.4 \%$ & $8,15.7 \%$ & \\
\hline Other $(n=4)$ & $2,6.3 \%$ & $2,2.9 \%$ & & 0 & $2,3.9 \%$ & \\
\hline Time interval (m) & $33.7 \pm 16.4$ & $25.3 \pm 16.1$ & 0.018 & $25.4 \pm 13.4$ & $28.4 \pm 17.1$ & 0.731 \\
\hline $\mathrm{hsCRP}(\mathrm{mg} / \mathrm{L})$ & $18.4 \pm 49.3(n=27)$ & $9.4 \pm 23.3(n=67)$ & 0.373 & $2.62 \pm 2.93(n=13)$ & $14.53 \pm 40.49(n=43)$ & 0.337 \\
\hline Low GFR $\left(<90 \mathrm{~mL} / \mathrm{min} / 1.73 \mathrm{~m}^{2}, \mathrm{n}\right)$ & $25 / 31,80.6 \%$ & $42 / 67,62.7 \%$ & 0.123 & $12 / 14,85.7 \%$ & $33 / 50,66.0 \%$ & 0.199 \\
\hline
\end{tabular}

SD, standard deviation; DM, diabetes mellitus; HL, hyperlipidemia; SVD, small vessel disease; LVD, large vessel disease; CE, cardioembolism; GFR, glomerular filtration rate.

Table 3. The factors associated with progression of white matter hyperintensities in ischemic stroke patients by a multiple logistic regression model

\begin{tabular}{lccc}
\hline & OR & $95 \% \mathrm{Cl}$ & $90 \% \mathrm{Cl}$ \\
\hline $\begin{array}{l}\text { Age } \\
<70\end{array}$ & & & \\
$\geq 70$ & 1.000 & & \\
Sex & 2.508 & $0.910-7.298$ & $1.056-5.958$ \\
$\mathrm{~F}$ & & & \\
M & 1.000 & & \\
Stroke type & 2.957 & $1.051-9.037$ & $1.207-7.242$ \\
$\quad$ Non-LVD & & & \\
LVD & 1.000 & & \\
GFR & 1.955 & $1.171-3.366$ & $1.261-3.031$ \\
$\quad$ Normal & & & \\
Abnormal $\left(<90 \mathrm{~mL} / \mathrm{min} / 1.73 \mathrm{~m}^{2}\right)$ & 1.000 & & \\
Dyslipidemia & 2.900 & $0.910-10.701$ & $1.045-8.046$ \\
$\quad$ No & & & \\
Yes & 1.000 & & \\
Smoking & 1.300 & $0.492-3.400$ & $0.578-2.925$ \\
$\quad$ No & & & \\
Yes & 1.000 & & \\
\hline
\end{tabular}

OR, odds ratio; $\mathrm{Cl}$, confidence interval; LVD, large vessel disease; GFR, glomerular filtration rate.

stroke patients.

As a novel finding of this study, 14 patients among 65 patients who had WMH on the initial scan showed regression. There have been two case reports that suggested the reversibility of cerebral white matter lesions in patients with carotid artery stenosis and with lacunar stroke. ${ }^{6,7} \mathrm{Up}$ to now, there has been no report about regression of WMH in ischemic stroke patients, even in normal elderly individuals. The neuropathologic substrate of WMH is a combination of perivascular demyelination, edema, gliosis, loss of axons, and glial cells accompanied by spongiosis. Alternative and mixed hypotheses for the pathogenesis of leukoaraiosis may account for the regression of $\mathrm{WMH} .{ }^{13}$ Disturbance in cerebrospinal fluid circulation and vasogenic cerebral edema, both of which are reversible conditions, may be interrelated to the ischemic origin of leukoaraiosis. There are previous case reports supporting this. Patients with hepatic encephalopathy showed regression of WMH with improvement of hepatic function. Certain transient medical illness associated with increased brain water such as hepatic encephalopathy may contribute to the reversibility of WMH. Although our patients did not have any hepatic illness, the condition of acute ischemic stroke state or other unidentified transient medical illness related with blood-brain-barrier disruption or edema might be present. Recent hypothesis suggested that patients with small vessel disease show leakage of toxic plasma components through an impaired blood-brain-barrier and cause secondary interstitial edema and injury to axons, myelin, and glial cell. ${ }^{14}$

With regard to the regression of WMH, we could not find any significant related factors. This is the first announcement of WMH regression, a further follow-up study with prospective observation is needed to confirm our results. Furthermore, serologic factors related to the blood brain barrier, cerebrospinal fluid circulation, and venous pressure can be considered in the investigation for a reversible mechanism.

In this study, thirty two percent of patients showed progression of white matter change. This is higher than the previously reported value, ${ }^{3}$ probably because the subjects in this study are all suffering from ischemic stroke who have many vascular risk factors compared with normal elderly individuals of previous 
studies.

Regarding factors associated with the progression of WMH, old age, male gender, large vessel disease, and decreased renal function were identified as significantly associated factors. Old age is a well-known risk factor for the development of WMH. Large vessel disease induces chronic cerebral ischemia. It seems that not focal ischemia such as small vessel stroke, but diffuse ischemia from large vascular insufficiency is responsible for the progression of WMH. This finding is consistent with a previous report suggesting that leukoaraiosis is related to the stroke subtype of large artery atherosclerosis. ${ }^{15}$ Poor renal function estimated by decreased GFR was significantly associated with WMH progression in this study. Renal function represents small-vessel pathology in the body. Leukoaraiosis is also from diffuse vascular insufficiency of the cerebral small vasculature. This result supports a recent report about renal function and leukoaraiosis in young stroke patients. ${ }^{16}$ The reason why current smoking is negatively associated with $\mathrm{WMH}$ progression is difficult to explain. It may be an incidental finding which needs further investigation.

As a limitation, our study enrolled a small number of subjects compared to previous cohort studies. However, the subjects were specified as ischemic stroke patients, which is a true reflection of real clinical practice. This may also be one of our study's assets. We used either T2-weighted MRI or FLAIR MRI for the evaluation of volume change. This might lessen the intra-case comparability. However, we used the same sequence to the initial scan for the exact comparison. There was no difference of the proportion of WMH changes between T2 and FLAIR MRI. Finally, the retrospective design of our study made the time intervals of the MRI scans irregular. Thus, we had to adjust the time interval in the multiple logistic regression models.

\section{Conclusions}

In $21.5 \%$ of ischemic stroke patients, white matter hyperintensities regressed on follow-up scan. Progression was observed in a third. For the documentation of the reversibile WMH in patients with ischemic stroke, we need further study to find associated factors and clinical significance and the way to reduce the burden of WMH.

\section{References}

1. de Leeuw FE, de Groot JC, Achten E, Oudkerk M, Ramos L, Heijboer R, et al. Prevalence of cerebral white matter lesions in elderly people: a population based magnetic resonance imaging study. The Rotterdam Scan Study. J Neurol Neurosurg Psychiatry 2001;70:9-14.
2. Debette S, Markus HS. The clinical importance of white matter hyperintensities on brain magnetic resonance imaging: systematic review and meta-analysis. BMJ 2010;341:c3666.

3. Enzinger C, Fazekas F, Ropele S, Schmidt R. Progression of cerebral white matter lesions -- clinical and radiological considerations. J Neurol Sci 2007;257:5-10.

4. Longstreth WT Jr, Arnold AM, Beauchamp NJ Jr, Manolio TA, Lefkowitz D, Jungreis C, et al. Incidence, manifestations, and predictors of worsening white matter on serial cranial magnetic resonance imaging in the elderly: the Cardiovascular Health Study. Stroke 2005;36:56-61.

5. Gouw AA, van der Flier WM, Fazekas F, van Straaten EC, Pantoni L, Poggesi A, et al. Progression of white matter hyperintensities and incidence of new lacunes over a 3-year period: the Leukoaraiosis and Disability study. Stroke 2008;39:1414-1420.

6. Yamada K, Sakai K, Owada K, Mineura K, Nishimura T. Cerebral white matter lesions may be partially reversible in patients with carotid artery stenosis. AJNR Am J Neuroradiol 2010;31: 1350-1352.

7. Durand-Birchenall J, Leclercq C, Daouk J, Monet P, Godefroy $\mathrm{O}$, Bugnicourt JM. Attenuation of brain white matter lesions after lacunar stroke. Int J Prev Med 2012;3:134-138.

8. Rovira A, Mínguez B, Aymerich FX, Jacas C, Huerga E, Córdoba J, et al. Decreased white matter lesion volume and improved cognitive function after liver transplantation. Hepatology 2007; 46:1485-1490.

9. Mínguez B, Rovira A, Alonso J, Córdoba J. Decrease in the volume of white matter lesions with improvement of hepatic encephalopathy. AJNR Am J Neuroradiol 2007;28:1499-1500.

10. Fazekas F, Chawluk JB, Alavi A, Hurtig HI, Zimmerman RA . MR signal abnormalities at $1.5 \mathrm{~T}$ in Alzheimer's dementia and normal aging. AJR Am J Roentgenol 1987;149:351-356.

11. Smith CD, Snowdon DA, Wang H, Markesbery WR. White matter volumes and periventricular white matter hyperintensities in aging and dementia. Neurology 2000;54:838-842.

12. Adams HP Jr, Bendixen BH, Kappelle LJ, Biller J, Love BB, Gordon DL, et al. Classification of subtype of acute ischemic stroke: definitions for use in a multicenter clinical trial. Trial of Org 10172 in Acute Stroke Treatment. Stroke 1993;24:35-41.

13. Pantoni L, Garcia JH. Pathogenesis of leukoaraiosis: a review. Stroke 1997;28:652-659.

14. Wardlaw JM, Sandercock PA, Dennis MS, Starr J. Is breakdown of the blood-brain barrier responsible for lacunar stroke, leukoaraiosis, and dementia?. Stroke 2003;34:806-812.

15. Lee SJ, Kim JS, Lee KS, An JY, Kim W, Kim YI, et al. The leukoaraiosis is more prevalent in the large artery atherosclerosis stroke subtype among Korean patients with ischemic stroke. BMC Neurol 2008;8:31. 
16. Steinicke R, Gaertner B, Grittner U, Schmidt W, Dichgans M, Heuschmann PU, et al. Kidney function and white matter dis- ease in young stroke patients: analysis of the stroke in young fabry patients study population. Stroke 2012;43:2382-2388. 